\title{
Expression and regulation of scavenger receptor class $B$ type I (SR-BI) in gall bladder epithelium
}

\author{
J F Miquel, M Moreno, L Amigo, H Molina, P Mardones, I I Wistuba, A Rigotti
}

Gut 2003;52:1017-1024

See end of article for authors' affiliations

\section{Correspondence to:}

J F Miquel and A Rigotti,

Departamento de

Gastroenterología,

Facultad de Medicina

Pontificia Universidad

Católica, Marcoleta 367,

Santiago, Chile;

jfmiquel@med.puc.cl.

Accepted for publication

8 January 2003
Background and aims: Biliary lipid absorption by the gall bladder mucosa and the cholesterol content of the gall bladder wall appear to play a role in cholesterol gall stone formation. As the scavenger receptor class B type I (SR-BI) regulates cellular cholesterol uptake, we studied its expression in human and murine gall bladders, its regulation by increased biliary lipid content, and its role in gall stone formation.

Methods and results: Using immunohistochemistry, SR-BI was found in the apical domain of human gall bladder epithelial cells. Immunoblotting of isolated membranes from gall bladder epithelial cells showed a specific signal for the 82 kDa SR-BI protein. In C57BL/6 mice, SR-BI was also found in the gall bladder epithelium. Using western blot analysis, an inverse relationship was observed between biliary cholesterol concentration and SR-BI expression in murine gall bladder mucosa. By comparing lithogenic diet fed wild-type and SR-BI deficient mice, gall bladder wall cholesterol content and gall stone formation were not found to be dependent on SR-BI expression.

Conclusions: (i) SR-BI is expressed in both human and murine gall bladder epithelium; (ii) biliary cholesterol hypersecretion is associated with decreased gall bladder SR-BI expression in mice; and (iii) murine SR-BI is not essential in controlling gall bladder wall cholesterol content and gall stone formation during diet induced cholelithiasis.
$\mathrm{T}$ he pathogenic mechanisms involved in cholesterol gall stone formation include both sustained hepatic secretion of cholesterol supersaturated bile as well as relevant downstream defects in the biliary tree such as gall bladder (GB) hypomotility and rapid GB bile cholesterol precipitation. ${ }^{1}$ Indirect ${ }^{2-7}$ and direct $^{8-11}$ experimental approaches in humans and animal models have suggested that the GB epithelium absorbs a significant fraction of biliary cholesterol. This phenomenon might have a significant effect on normal processes (for example, GB biliary lipid composition, body cholesterol metabolism) and also pathophysiological conditions. ${ }^{11}{ }^{12}$ With regard to cholesterol gall stone formation, a decreased lipid absorptive function of the GB could enhance cholesterol supersaturation of hepatic bile facilitating cholesterol precipitation. ${ }^{11-13}$ On the other hand, a higher cholesterol absorption through the GB epithelium could determine an increased content of cholesterol in GB muscle cells, resulting in impaired GB contraction (hypomotility) thereby contributing further to gall stone formation..$^{14} 15$

Despite the evidence indicating that the GB epithelium absorbs biliary cholesterol, the underlying molecular mechanisms and dynamics involved in cholesterol absorption by the GB epithelium remain unknown. ${ }^{12}{ }^{13}$ However, recent studies have indicated that cholesterol absorption in the intestinal epithelium may be a protein regulated process. ${ }^{16-18}$ In fact, the importance of ATP binding cassette (ABC) transporters, such as ABCG5 and ABCG8, in modulating intestinal cholesterol absorption has been recently established. ${ }^{19}$ Another potential candidate for regulation of transepithelial cholesterol transport is the scavenger receptor class B type I (SR-BI). SR-BI was initially identified as a cell surface multilipoprotein receptor involved in the selective uptake of lipoprotein cholesterol, mainly in the liver and steroidogenic tissues. ${ }^{20-23}$ Furthermore, SR-BI can also mediate cellular uptake of non-lipoprotein cholesterol as well as free cholesterol efflux from cells to extracellular acceptors (reviewed by Rigotti and colleagues ${ }^{21}$ and Kriege ${ }^{22}{ }^{23}$ ). Interestingly, SR-BI is expressed in the brush border membrane of the small intestine, ${ }^{24-26}$ and in vitro chol- esterol uptake assays using intestinal epithelial cells suggested its role in cholesterol absorption. ${ }^{24}$ In addition, changes in intestinal SR-BI expression correlated with variations in cholesterol absorption rate in rodents. ${ }^{25}$ However, murine SR-BI deficiency was associated with a subtle increase or no change in intestinal cholesterol absorption, indicating that SR-BI expression is not essential for this process. ${ }^{26}{ }^{27}$

Similar to intestinal cholesterol absorption, it can also be postulated that cholesterol transport through the GB epithelium is a protein mediated process. ${ }^{11-13}$ During the preparation of this manuscript, SR-BI expression in the human GB was reported.$^{28}$ In the present study, we also show that SR-BI is highly expressed in the apical domain of epithelial cells from human and murine GB. In addition, we demonstrate that dietary manipulations that increase the GB bile cholesterol content are associated with a marked decrease in expression of SR-BI in the murine GB. However, SR-BI expression is not essential in controlling either GB wall cholesterol content or gall stone formation in mice fed a lithogenic diet.

\section{MATERIALS AND METHODS}

Human GB samples and isolation of GB epithelial cells Human GB was obtained by elective laparoscopic cholecystectomy performed for cholesterol gall stone disease. None of the patients had a previous or current clinical history of acute cholecystitis. Tissue sections of freshly excised GB were fixed in formalin, embedded in paraffin, sectioned at $5 \mu \mathrm{m}$, and stained with haematoxylin and eosin (HEE) using standard histological procedures. Some freshly excised GBs were immediately transported to the laboratory at $4^{\circ} \mathrm{C}$ in sterile

Abbreviations: $A B C$, ATP binding cassette; $A P N$, aminopeptidase- $N$; DMEM, Dulbecco's modified Eagle's medium; GB, gall bladder; GBEC gall bladder epithelial cell; H\&E, haematoxylin-eosin; SDS-PAGE, sodium dodecyl sulphate-polyarylamide gel electrophoresis; SR-BI, scavenger receptor class $B$ type I. 
medium consisting of a 1:1 (v/v) mixture of Dulbecco's modified Eagle's medium (DMEM) and Ham's F12 (Life Technologies, Grand Island, New York, USA) containing antibiotics, as described by Auth et al with minor modifications. ${ }^{27}$ Isolation of human GB epithelial cells (GBEC) was performed between 30 minutes and one hour after surgery. The GB was incised longitudinally, the walls were reflected, and a small section of tissue was taken for histological analysis. Then, the mucosa was rinsed carefully with transport medium and wiped with gauze several times to remove adherent bile and mucus. The GB tunica mucosa was then placed in $0.125 \%$ collagenase solution (collagenase type IV; Sigma Chemicals, St Louis, Missouri, USA) for 20 minutes at $37^{\circ} \mathrm{C}$. Every five minutes the mucosa was abraded thoroughly using a scalpel and flushed with DMEM medium. The resulting cell suspension was subjected twice to centrifugation at $85 \mathrm{~g}$ for five minutes at $20^{\circ} \mathrm{C}$. An aliquot of freshly isolated GBEC was placed on a glass slide, fixed with ethanol, and stained with H\&E for light microscopy. Semiquantitative analysis demonstrated that more than 95\% of cells had epithelial features. GBEC were kept at $-80^{\circ} \mathrm{C}$ for further western blot analysis, as described below.

\section{Animals and diets}

C57BL/6 mice, purchased originally from the Jackson Laboratory (Bar Harbour, Maine, USA), were used to breed our own colony. Animals were housed in a humidity and temperature controlled room with reverse cycle lighting. All mice were maintained with a water and chow diet $(<0.02 \%(\mathrm{w} / \mathrm{w})$ cholesterol; Prolab RMH3000, PMI Feeds Inc, St Louis, Missouri, USA) ad libitum, prior to the feeding experiments with cholesterol rich or diosgenin containing diets. Male C57BL/6 mice (two months old) were fed chow diet, or were switched as indicated to a high cholesterol diet (chow diet supplemented with $2 \%(\mathrm{w} / \mathrm{w})$ cholesterol) or to a lithogenic diet of high cholesterol/high fat/bile acid content (1.25\% cholesterol, 15\% total fat, $0.5 \%$ cholic acid; TD9022 1, Harlan Teklad, Madison, Wisconsin, USA). Groups of at least four animals each were subjected to intervals of 3,6,10, 12, and 30 days of dietary manipulation. An additional group of mice were fed chow diet or chow supplemented with $1 \%(\mathrm{w} / \mathrm{w})$ diosgenin for two weeks.

To evaluate the role of SR-BI expression in controlling cholesterol content of the GB wall and gall stone formation, mice with a targeted mutation in the $s r-b i$ locus $^{26}$ were initially obtained from Dr Monty Krieger (Massachusetts Institute of Technology, Cambridge, Massachusetts, USA). The mutation in the $s r-b i$ gene was maintained in a mixed genetic background $(\mathrm{C} 57 \mathrm{BL} / 6 \times 129 / \mathrm{Sv})$ by crossing heterozygous $s r-b i$ mutant female and male mice. Homozygous SR-BI knockout mice were screened by polymerase chain reaction. Male SR-BI knockout mice (2-3 months old) as well as sex and age matched control mice were studied under chow or lithogenic diets, as described above.

All protocols were carried out according to accepted criteria for humane care of experimental animals and approved by the Review Board for Animal Studies of our institution.

\section{Collection of GB bile and tissues in mice}

Surgery was performed on mice that were fasted overnight with free access to water. Animals were anaesthetised with an intraperitoneal injection of pentobarbital $(4.5 \mathrm{mg} / 100 \mathrm{~g}$ body weight) and the abdominal cavity was exposed through a ventral incision; the cystic duct was ligated and the GB removed. Mice were then killed and the liver and $15 \mathrm{~cm}$ of the proximal jejunum were removed. The GB was examined visually for the presence of stones or sediment. Bile was aspirated by puncturing the GB with a fine needle and then stored at $-20^{\circ} \mathrm{C}$ for further lipid analysis. ${ }^{30} \mathrm{GBs}$ from animals of the same experimental group $(n=4-5)$ were opened longitudinally, washed with phosphate buffered saline, and pooled. In some animals, following aspiration of bile as described, ethanol (200-300 $\mu \mathrm{l})$ was instilled into the GB lumen before fixing by immersion in $95 \%$ ethanol, embedding in paraffin, sectioning, and staining with H\&E for histological examination or antibodies for immunohistochemistry. Segments of jejunum were washed with buffer and everted. Jejunal mucosal scrapings were obtained and pooled $(n=4)$ from animals of the same experimental group. GB, jejunal, and liver samples were stored at $-80^{\circ} \mathrm{C}$ for further analysis.

\section{Antibodies}

Rabbit antiserum against human SR-BI, which cross reacts with murine SR-BI, was obtained from Dr Susan Acton (Millennium Pharmaceuticals Inc., Cambridge, Massachusetts, USA). A rabbit polyclonal antibody raised against rat aminopeptidase-N (APN 1637-5; kindly provided by Dr Ann Hubbard, Johns Hopkins Medical School, Baltimore, Maryland, USA) was used in western blots to normalise the signal generated by the anti-SR-BI antibody. APN is a well known membrane bound metalloprotease expressed in the apical domains of hepatocytes and epithelial cells of the small intestine, kidney, and biliary tract. ${ }^{31} 32$

\section{Immunohistochemistry}

Tissue sections were deparaffinised and endogenous peroxidases were quenched with $0.3 \% \mathrm{H}_{2} \mathrm{O}_{2}$ in methanol for $30 \mathrm{~min}$ utes at room temperature. Immunostaining was performed in a Nexes IHC staining system (Ventana Medical System, Inc., Tucson, Arizona, USA). Incubation with the primary antibody was carried out for 90 minutes in appropriate dilutions (antiSR-BI 1:300). The specific signal was developed with an universal biotinylated secondary antibody and peroxidase activity was revealed with 3,3'- diaminobenzidine hydrochloride and $\mathrm{H}_{2} \mathrm{O}_{2}$, according to the reagents and instructions of the manufacturer's detection system. Subsequently, sections were counterstained lightly with H\&E, dehydrated, cleared, and mounted in Permount. Negative controls were obtained by omitting the primary antibody or by incubating sections with isotypic immunoglobulins.

\section{Immunoblotting}

Crude membranes were prepared from isolated human GBEC, pools of murine GB and jejunal epithelium, and murine liver, as described elsewhere. ${ }^{33}$ Each sample ( $20 \mu \mathrm{g}$ total membrane protein) was subjected to $10 \%$ sodium dodecyl sulphatepolyarylamide gel electrophoresis (SDS-PAGE) under reducing conditions and transferred to Hybond $\mathrm{C}$ membranes (Amersham, Arlington Heights, Illinois, USA). The blots were incubated with anti-SR-BI (1:3000 dilution) or anti-APN (1:400 dilution) polyclonal antibodies in Tris buffered saline/0.05\% Tween 20 containing 5\% non-fat dried milk powder. Visualisation of membrane bound antibodies was carried out with a standard chemiluminescence kit (Amersham) using horseradish peroxidase labelled mouse antirabbit IgG antibody (Sigma Chemicals) as a secondary antibody. Western blot signals were quantified by densitometric analysis (CS-9000 Dual-Wavelength Flying-Spot Scanner, Shimadzu), and results were normalised to the signal generated by APN.

\section{Analytical procedures}

Protein concentrations were determined with the Bradford assay, using bovine serum albumin for the standard curve. Biliary bile salts were determined by the $3 \alpha$-hydroxysteroid dehydrogenase method, lecithin by the inorganic phosphorous procedure, and cholesterol by the cholesterol oxidase assay, as described previously. Unesterified and esterified cholesterol content was measured after lipid extraction ${ }^{34}{ }^{35}$ in pools of GB samples $(n=4-5)$ obtained from mice fed with chow or the lithogenic diet. 

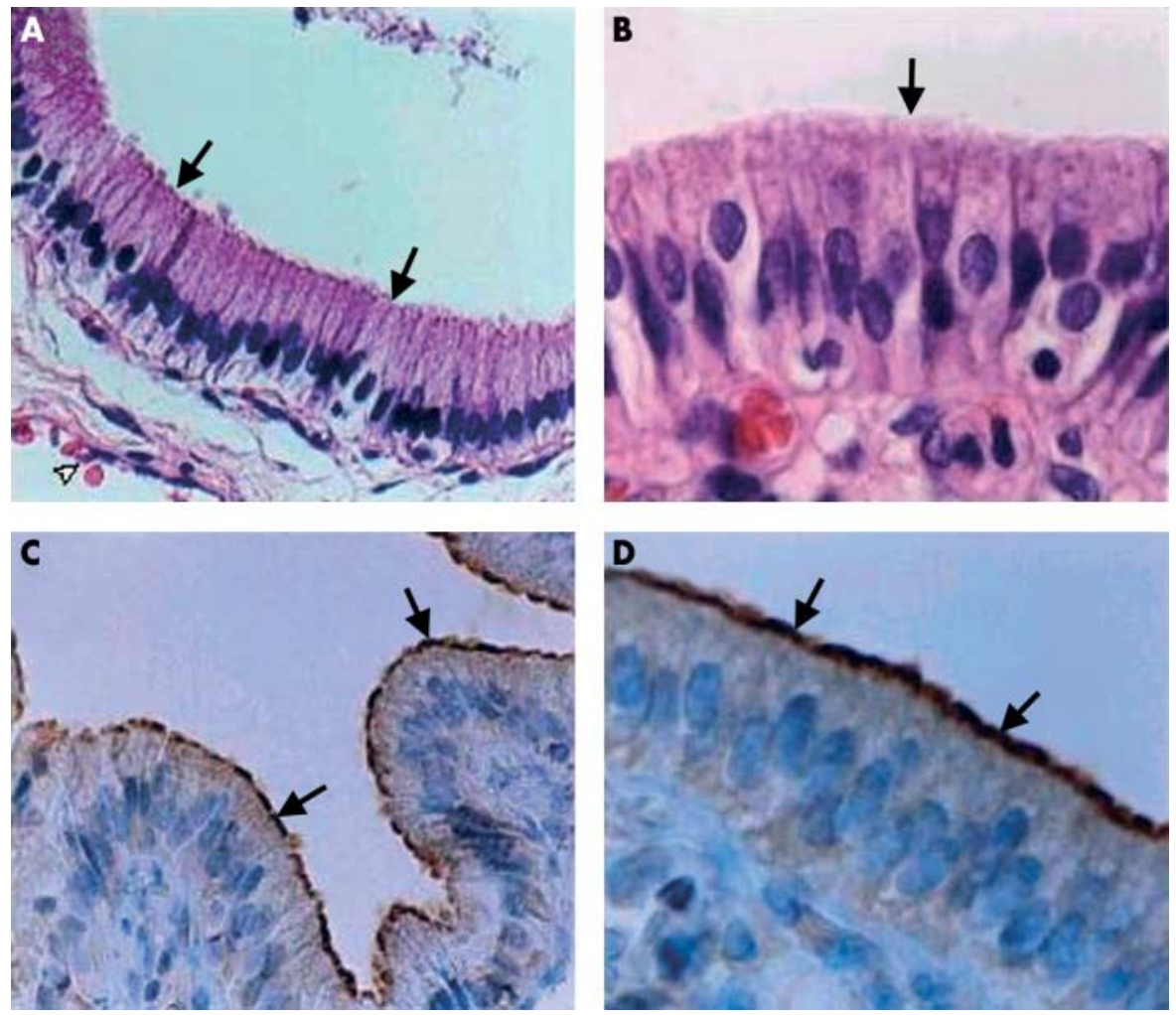

Figure 1 Haematoxylin-eosin (H\&E) staining $(A, B)$ and immunohistochemical reaction for scavenger receptor class $B$ type I (SR-BI) (C, D) in human gall bladder (GB) epithelium. Immunohistochemistry (performed as described in materials and methods) is based on a polyclonal anti-SR-BI as the primary antibody. (A, B) H\&E staining revealed a well preserved monolayer of typical $G B$ epithelial cells with an apical domain (arrows), and subepithelial capillary vessels (open arrowhead). (C, D) Strong immunoperoxidase reaction for SR-BI protein was restricted to the plasma membrane of the apical domain of the GB epithelial cells (arrows). No staining was observed in the basolateral domain or in the tissues of the lamina propria. Original magnification: $A$ and $C, 200 x ; B$ and $D, 400 x$.

\section{Statistical analysis}

Values are expressed as mean (SEM). The significance of differences between means was evaluated using the unpaired Student's $t$ test. The level of significance was set at $\mathrm{p}<0.05$.

\section{RESULTS}

\section{SR-BI is expressed in the apical domain of human and} murine GB epithelial cells

Human GBs were obtained from patients with cholesterol gall stone disease after elective cholecystectomy and immunohistochemistry was performed to assess whether the scavenger receptor SR-BI was expressed in the GB epithelium. Routine H\&E staining of the GB showed a well preserved monolayer of GBEC with a mild degree of chronic cholecystitis (fig 1A, 1B). Immunohistochemical staining of GBs showed a clear signal for the anti-SR-BI antibody restricted to the apical pole (brush border membrane) of the GBEC (fig IC, ID, see arrows). No reaction was detected in GBEC when the first antibody was omitted (data not shown).

In order to confirm whether the positive immunostaining in the GB corresponded to the well characterised SR-BI protein, ${ }^{20}$ GBEC were isolated from freshly harvested human GB and total cell membranes were prepared and subjected to immunoblot analysis. Figure 2 (left lane) shows the presence of the $\approx 82 \mathrm{kDa}$ SR-BI protein in the total membrane fraction of human GBEC. Detection of the expected molecular weight of mature SR-BI protein in our GB samples is in clear contrast with the $57 \mathrm{kDa}$ SR-BI protein recently reported in human GB (Johnson and colleagues, ${ }^{28}$ see discussion). It is noteworthy that in our GB samples, SR-BI expression levels were similar to those observed in a total membrane preparation from mouse liver (fig 2, right lane).

To evaluate whether SR-BI was also expressed in the GBEC of $\mathrm{C} 57 \mathrm{BL} / 6$ mice, a well defined animal model used for diet

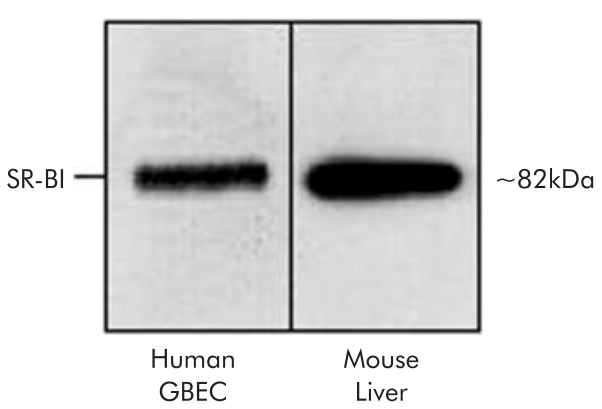

Figure 2 Immunoblot analysis of scavenger receptor class B type I (SR-BI) in crude membrane fractions from isolated human gall bladder epithelial cells (GBEC) and from male murine liver (chow diet). GBECs were harvested from a fresh human GB without cholesterolosis, and crude plasma membranes were isolated as described in materials and methods. Liver from a control C57BL/6 mouse was used for isolation of crude plasma membrane. Crude membranes (20 $\mu \mathrm{g}$ protein each) were subjected to sodium dodecyl sulphate-polyarylamide gel electrophoresis and electrophoretically transferred to a nitrocellulose filter. Immunoblot analysis was carried out using anti-human SR-BI polyclonal antibody. Bound antibodies were visualised as described in materials and methods.

induced cholesterol gall stone disease, ${ }^{30} \mathrm{~GB}$ of chow fed mice were also subjected to immunohistochemical analysis. Following ethanol fixation, H\&E staining showed a well preserved monolayer of murine GBEC ( fig 3A, 3B) with identifiable apical (black arrows) and basal (white arrowheads) plasma membrane domains. Immunohistochemical analyses of murine GB with the anti-SR-BI serum are shown in figs $3 C$ and 3D. Positive staining for SR-BI was restricted to the apical pole of the GBEC (arrows), similar to the signal found in human GB (fig IC, ID). 

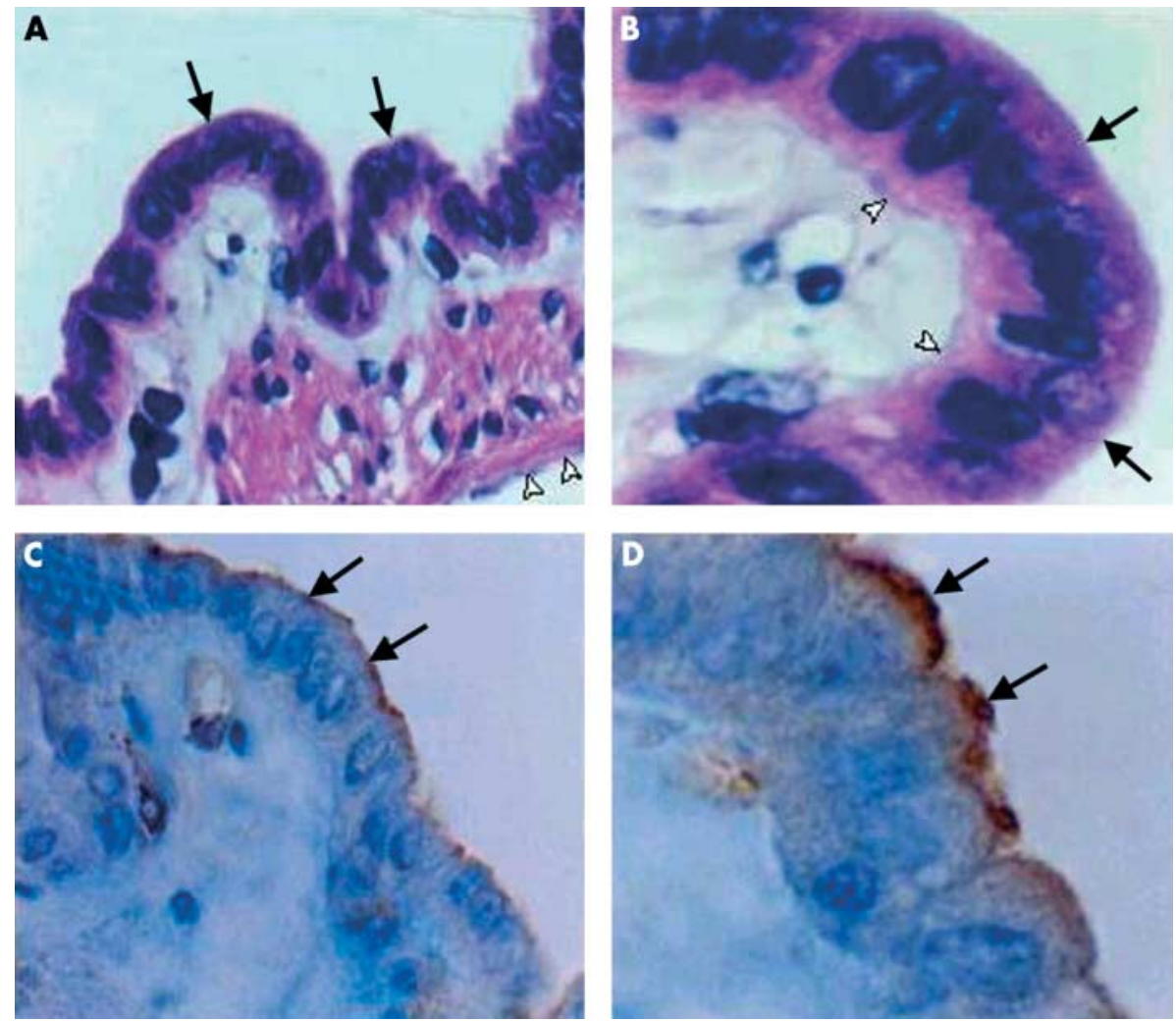

Figure 3 Haematoxylin-eosin staining (A, B) and immunohistochemical localisation of scavenger receptor class $B$ type I (SR-BI) (C, D) in gall bladder (GB) epithelium of mice on a chow diet. Immunohistochemistry is based on a polyclonal anti-SR-Bl as the primary antibody. (A, B) The section shows GB epithelial cells and their apical domain (arrows), together with tissues of the lamina propria and tunica serosa (open arrowhead). (C, D) A clear signal for SR-BI protein is restricted to the plasma membrane in the apical domain of the GB epithelium (arrows); no staining is observed in the basolateral domain, or in the subepithelial tissues. Original magnification: A and C, 200x; B and D, 400x.

Table 1 Lipid composition of gall bladder bile from C57BL/6J mice fed a control diet, a diet supplemented with $2 \%$ cholesterol, or a lithogenic diet

\begin{tabular}{lclcc}
\hline Group & $\begin{array}{l}\text { Cholesterol } \\
(\mathrm{mmol} / \mathrm{l})\end{array}$ & $\begin{array}{l}\text { Phospholipids } \\
(\mathrm{mmol} / \mathrm{l})\end{array}$ & Bile salts (mmol/l) & $\begin{array}{l}\text { Gall stones or } \\
\text { sludge (\%) }\end{array}$ \\
\hline Control & $1.84(0.2)$ & $14.3(1.7)$ & $84.4(8.3)$ & 0 \\
$2 \%$ Cholesterol & $5.05(0.5)^{*}$ & $14.2(1.2)$ & $131.1(10.7)^{*}$ & 0 \\
Lithogenic & $11.67(0.4)^{*}$ & $44.4(1.1)^{*}$ & $227.5(16.4)^{*}$ & 100 \\
\hline
\end{tabular}

Mice ( $n=4$ for each group) were fed the different experimental diets for four weeks.

* Significantly different from the two other groups $(p<0.005)$.

\section{GB SR-BI expression is downregulated in association with an increased biliary cholesterol content in \\ C57BL/6 mice}

The next part of the study was designed to determine whether expression of SR-BI in the GBEC could be modulated under conditions of increased GB biliary lipid content induced by dietary manipulations in mice. C57BL/6 mice were first fed for four weeks with a control diet, a diet supplemented with $2 \%$ cholesterol, or a high cholesterol/fat/bile salt lithogenic diet. As shown in table 1, GB biliary cholesterol and bile salts were increased significantly (by 2.7-fold and 1.5-fold, respectively) in animals fed the high cholesterol diet for four weeks compared with animals on the control diet. As we have shown previously, ${ }^{30}$ the increment in GB biliary lipids in animals fed a lithogenic diet for four weeks was even more dramatic. Compared with the control diet, GB biliary cholesterol, phospholipid, and bile salt concentrations increased by 6-, 3-, and 2.7-fold, respectively. All mice developed biliary sludge or gall stones when fed the lithogenic diet.

Figure 4 shows SR-BI protein expression from murine GB and proximal jejunum epithelia under the different experi- mental diets. It is worth noting that the level of expression of SR-BI in murine GB epithelia under the control diet was similar to the protein expression level in the liver (fig 2), and significantly higher than that in the proximal gut. It was evident that under either the cholesterol supplemented or lithogenic diet for four weeks, protein expression levels of SR-BI in the GB decreased dramatically, by $30 \%$ and $80 \%$, respectively. In proximal intestine samples obtained from the same animals, only a low signal for SR-BI was visible, which was insufficient for quantifying differences in the level of intestinal SR-BI expression under the various experimental conditions.

Next we tested whether GB SR-BI expression could be regulated specifically in association with biliary cholesterol, but not bile salt, hypersecretion and whether downregulation of SR-BI in GB epithelium occurs before gall stone formation. The new experiment considered feeding of three groups of mice $(n=4)$ for only 10 days, under the same experimental conditions and diets as described above, and an additional group of mice fed either with chow or chow supplemented with diosgenin $(1 \% \mathrm{w} / \mathrm{w})$ for 14 days. After 10 days, the lithogenic diet induced a 4- to 5-fold increase in GB biliary 


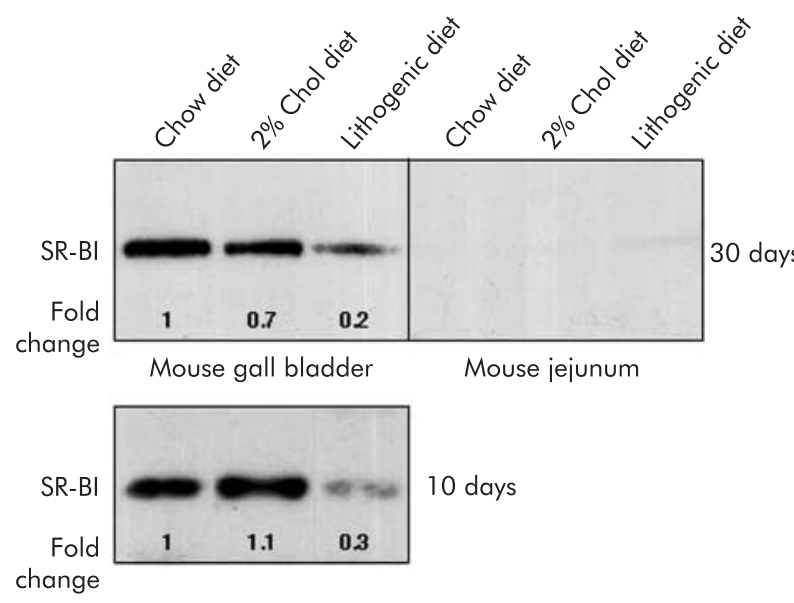

Figure 4 Immunoblot analysis of scavenger receptor class B type I (SR-BI) in crude membrane fractions from gall bladder (GB) and proximal jejunum epithelia of $\mathrm{C} 57 \mathrm{BL} / 6$ male mice fed a diet of chow alone, chow supplemented with $2 \%$ cholesterol (Chol), or a lithogenic diet for four weeks or 10 days. Epithelium from GBs and jejuna from each group of mice were pooled ( $n=4$ each), crude membranes were prepared, and aliquots (20 $\mathrm{\mu g}$ protein) were subjected to sodium dodecyl sulphate-polyarylamide gel electrophoresis analysis and transferred electrophoretically to a nitrocellulose filter, as described in the experimental procedures. Immunoblot analysis was carried out with rabbit anti-SR-BI as the primary antibody and bound antibodies were visualised as described in materials and methods. The degree of changes in SR-BI protein levels was compared with those of mice on the chow diet and are representative of two independent experiments. Quantitation of bands was determined after correction for loading differences with APN, as described in materials and methods.

cholesterol concentrations (1.08 (0.16) $v 4.94(0.86) \mathrm{mmol} / \mathrm{l}$ for control and lithogenic diets, respectively), without significant changes in bile salt or phospholipid content. ${ }^{30}$ Mice fed with $2 \%$ cholesterol showed no significant changes in GB biliary lipid composition after 10 days. Using polarised light microscopy, some cholesterol crystals, but not stones, were observed in GB bile of mice on the lithogenic diet. As previously described in rodents, ${ }^{36}$ diosgenin feeding increased GB biliary cholesterol content by 2.5 -fold in C57BL6 mice ( 1.0 (0.2) for chow fed mice v. $2.45(0.3) \mathrm{mM}$ for diosgenin fed mice).

Pooled GB tissues of these cholesterol and diosgenin fed mice were subjected to western blot analysis, as described above, and protein expression levels for SR-BI and APN were quantified. After 10 days on the lithogenic diet, a 70\% decrease in the expression level of SR- BI was observed. No changes in SR-BI expression were apparent after 10 days of feeding with the diet supplemented with cholesterol alone (fig 4). A 60\% decrease in the expression level of SR-BI in the GB of diosgenin fed mice was also observed (data not shown). Taken together, these results indicate that increased GB biliary cholesterol content per se (without associated changes in any other biliary lipid components) correlated with decreased GB SR-BI expression in mice.

\section{Lack of correlation between GB wall cholesterol content, gall stone formation, and SR-BI expression levels}

Different animal models of cholesterol gall stone disease have demonstrated that a lithogenic diet induces an increment in the cholesterol content of the GB smooth muscle plasma membrane, generating a contractile defect of this organ leading to an increased residence time of lithogenic bile in the GB lumen contributing further to gall stone formation and growth. ${ }^{14}{ }^{15}$ This excess of GB smooth muscle cholesterol may originate from the lithogenic GB bile itself..$^{6-13}$ To explore if
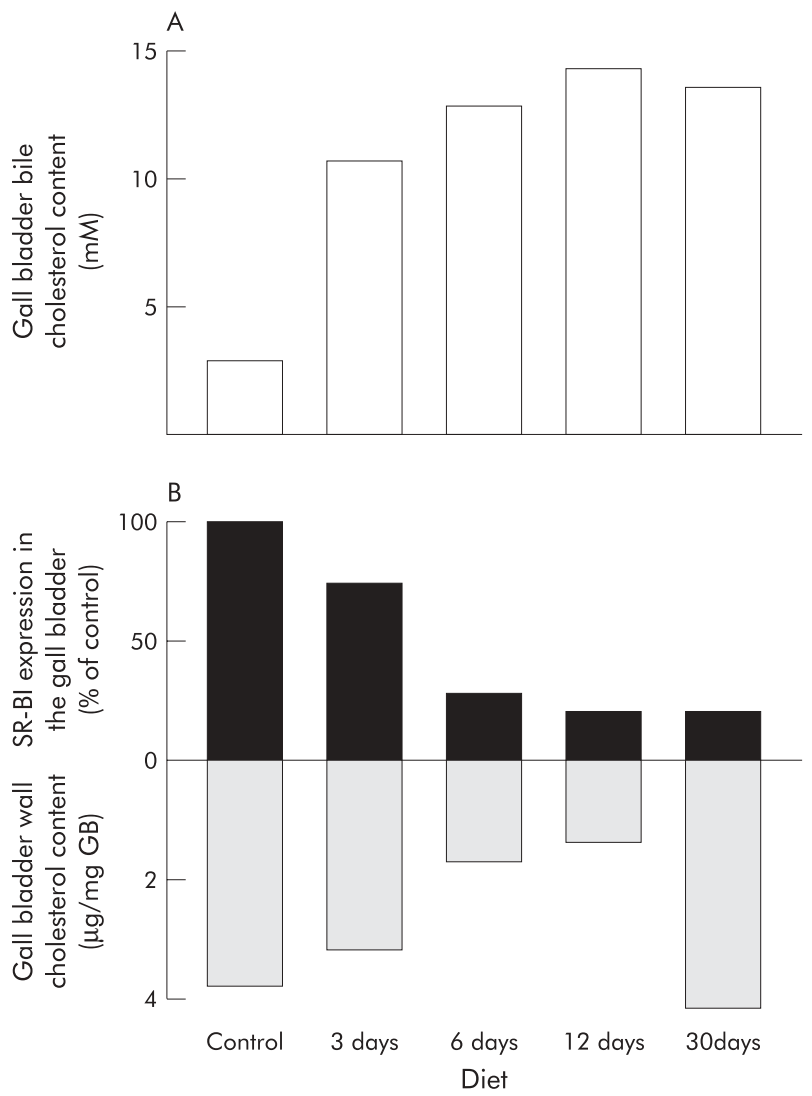

Figure 5 Correlation between gall bladder (GB) bile cholesterol content, GB wall total cholesterol content, and scavenger receptor class $B$ type I (SR-BI) expression on administration of either a chow or lithogenic diet. C57BL/6 mice were fed with a control or lithogenic diet for $3,6,12$, or 30 days. Total cholesterol content of GB bile (A), protein expression of SR-BI in the GB wall, and total cholesterol content of $G B$ tissue (B) were assessed as described in materials and methods over 30 days of diet administration. Total cholesterol content in bile increased progressively over time and correlated inversely with expression of SR-BI in the GB wall. Total cholesterol content of GB tissue correlated directly with SR-BI expression only during the first 12 days of administration of a lithogenic diet.

SR-BI was relevant in controlling the cholesterol content of the GB wall under conditions that dramatically increased biliary cholesterol concentration, we correlated GB SR-BI expression levels with cholesterol content in the GB wall C57BL/6 mice were sacrificed 3, 6, 12, and 30 days after feeding with chow or lithogenic diets. At each time point, GB bile and pooled GB walls were obtained for lipid analysis and cholesterol content, respectively. As shown in fig 5, increased biliary cholesterol content was associated with decreased GB SR-BI expression as early as three days after feeding a lithogenic diet. Even lower SR-BI expression levels (30\% of chow fed control mice) were maintained under lithogenic diet feeding between day 6 and 30. While a direct relationship was observed between GB SR-BI expression levels and cholesterol content during the first 12 days using the lithogenic diet, this correlation was lost after feeding this diet for 30 days (fig 5). These findings suggest that lowered SR-BI expression is not a sufficient event for reducing GB wall cholesterol content in lithogenic diet fed mice.

To further address the relevance of SR-BI expression in controlling GB wall cholesterol content and gall stone formation, mice with SR-BI deficiency in a mixed C57BL/6×129/SV background were studied. As shown in fig 6, GB cholesterol content of SR-BI knockout mice was similar to wild-type animals when fed a chow diet. After feeding the lithogenic diet 


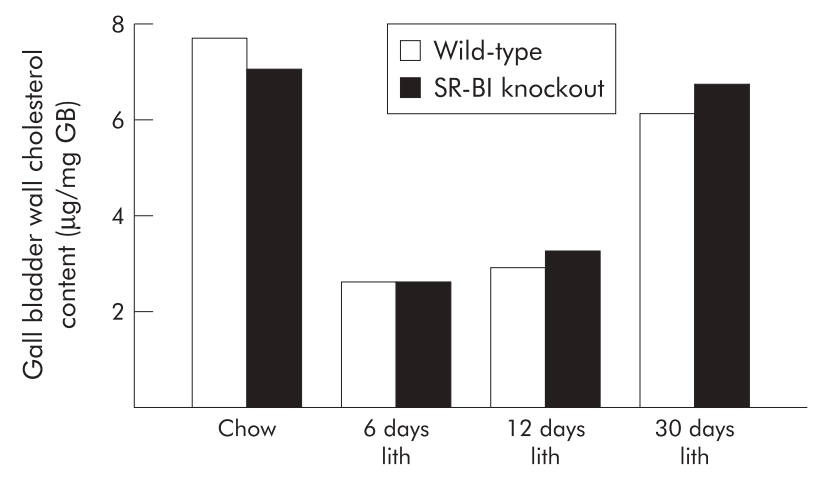

Figure 6 Total cholesterol content of gall bladder (GB) wall in wild-type and scavenger receptor class B type I (SR-BI) knockout mice on administration of either chow or a lithogenic (lith) diet for 6 12 , and 30 days. No differences were observed in total cholesterol content of the GB wall between wild-type and SR-BI null mice fed with either type of diet for the indicated periods. In both experimental groups, feeding a lithogenic diet elicited a biphasic response with a marked decrease in total cholesterol content of the GB wall during the first 12 days of diet administration which returned to control values after 30 days, similar to the response seen in $\mathrm{C} 57 \mathrm{BL} / 6$ mice (see fig 5).

for seven and 30 days, GB biliary cholesterol content was slightly, but not significantly, lower in SR-BI knockout mice compared with wild-type mice $(2.9$ (0.3) v 4.8 (1.9) $\mathrm{mM}, 3.8$ (1.2) $v 4.6$ (1.8) $\mathrm{mM}$, respectively; p>0.05). Furthermore, changes in GB wall cholesterol content of lithogenic diet fed SR-BI knockout mice were similar to those found in wild-type mice after feeding the same diet for 6, 12, or 30 days (fig 6). In addition, cumulative gall stone prevalence in SR-BI knockout mice after 30 days under a lithogenic diet was similar to that of wild-type mice ( 90 and $100 \%, n=10$ and 12 , respectively). These results indicate that SR-BI expression in the GB is not essential for controlling either GB cholesterol content or the development of cholesterol gall stones under a lithogenic diet.

\section{DISCUSSION}

In this study, we have demonstrated that SR-BI is highly expressed in GB epithelial cells. Immunohistochemical analysis of human and murine GB localises SR-BI to the apical domain of GBEC. SR-BI was markedly downregulated in mouse GBEC in association with increasing GB biliary cholesterol content. Finally, SR-BI expression was not essential in controlling either total GB wall cholesterol content under basal or lithogenic conditions or the incidence of gall stone formation.

SR-BI was initially discovered as a new class of receptor for modified lipoproteins. Later it was shown to be the first molecularly well defined cell surface high density lipoprotein receptor to be identified.$^{20}$ Interestingly, SR-BI mediates a cellular process known as selective lipid uptake by which not only cholesteryl esters, but also various other lipid molecules (that is, free cholesterol, phospholipids) from receptor bound lipoproteins are directly transferred to the cell plasma membrane (reviewed by Rigotti and colleagues ${ }^{21}$ and Krieger $^{22}$ ) Furthermore, SR-BI can also facilitate both non-lipoprotein unesterified cholesterol uptake as well as free cholesterol efflux in cultured cells. Various genetic manipulations in vivo have established that SR-BI plays important physiological and pathophysiological roles in lipoprotein cholesterol metabolism in mice..$^{22} 37$ More recently, SR-BI was proposed to play a role in intestinal cholesterol absorption based on SR-BI expression analysis and transport studies with intestinal brush border membranes and cells. ${ }^{24}{ }^{25}$ Several studies have shown that SR-BI is indeed expressed in the apical membrane of enterocytes of rats and mice, mainly in proximal segments of the gut where cholesterol absorption take place. ${ }^{25}$
More interestingly, Hauser et al used SR-BI ligands and anti-SR-BI antibodies to inhibit cholesterol uptake from bile salt mixed micelles and phospholipid vesicles into intestinal brush border preparations and CaCo 2 cells. ${ }^{24}$ However, further studies aimed at evaluating more directly the potential role of SR-BI in intestinal cholesterol absorption in SR-BI knockout mice established that expression of SR-BI is not essential for this process. ${ }^{26} 27$

Several studies have reported indirect evidence that GB cholesterol absorption may occur in vivo. In vitro experiments have shown that cholesterol is indeed efficiently absorbed by human GB mucosa. ${ }^{8-10}$ A recent study by Corradini et al, using an intraarterially perfused GB model, confirmed that the mucosa of normal human and pig GB is able to absorb $20-30 \%$ of biliary cholesterol over a period of five hours. ${ }^{11}$ As $800-1200 \mathrm{mg}$ of free cholesterol are secreted daily from the liver into the biliary tract in humans, ${ }^{38}$ absorption of at least $20 \%$ of biliary cholesterol by the GB could be similar to the daily amount of dietary cholesterol absorbed in the intestine in subjects on a typical Western diet. ${ }^{16}$ This significant amount of biliary cholesterol reabsorption in the GB may have major implications for biliary cholesterol solubility, intestinal cholesterol absorption, and, ultimately, for body cholesterol homeostasis. In fact, this function is impaired in the GB of cholesterol gall stone patients suggesting that lack of this absorptive capacity may contribute to gall stone formation. ${ }^{11}{ }^{13}$ Despite the potential physiological and pathophysiological relevance of GB function in cholesterol absorption, the detailed cellular and molecular mechanism(s) by which biliary lipids are actually absorbed by the GB epithelium remains unknown. A recent study has reported that apoliprotein (apo) A-I, a normal component of human bile, ${ }^{39}$ stimulated cholesterol transfer from phospholipid vesicles on the mucosal side to the serosal side of cultured polarised GBEC. In addition, APF, an amphipatic anionic polypeptide that exhibits immunological cross reactivity with apo A-I, ${ }^{40}$ could also serve as a protein ligand for candidate apical GBEC receptors, which might be involved in GB cholesterol absorption. In this regard, the previously reported functional properties of SR-BI and the enterocyte cholesterol transport inhibition experiments together with its high level of expression in GBEC and its localisation in the apical domain of these cells, as shown in this study, suggested a potential role of this cell surface receptor in GB cholesterol absorption.

While our western blot analysis showed the presence of a $82 \mathrm{kDa}$ SR-BI protein in human and murine GB, a previous study has reported an immunoreactive SR-BI protein of 57 $\mathrm{kDa}$, but not $82 \mathrm{kDa}$, in murine $\mathrm{GB} .{ }^{44} \mathrm{It}$ is interesting that this latter study also showed that most of the hepatic and ovarian protein detected by the anti-SR-BI antibodies exhibited a $57 \mathrm{kDa}$ molecular weight. Johnson et al suggested that this lower molecular weight SR-BI protein represented a deficiently glycosylated form due to slow maturation of SR-BI and/or increased turnover of mature SR-BI. The discrepancy between our work and that of Johnson et al requires further study. However, we speculate that it may be due to mouse strain and/or antibody specificity differences or may be potentially explained by SR-BI protein deglycosylation during GB tissue processing for immunoblotting.

Another important finding of this study was the dramatic downregulation of GB SR-BI expression under conditions of high biliary lipid content. Cholesterol feeding either in a high cholesterol diet or a high cholesterol/high fat/cholic acid lithogenic diet for four weeks resulted in lowered expression of SR-BI in murine GB (fig 4, 5), which correlated with an increased concentration of GB biliary lipids (table 1). Both the $2 \%$ cholesterol diet and the cholesterol rich lithogenic diet produced not only a dramatic increase in GB bile cholesterol concentration but also a significant increase in biliary phospholipids and/or bile salts. Therefore, this in vivo experimental model did not allow identification of exactly which increase in biliary lipid content was correlated with regulation 
of SR-BI expression in GB epithelia. To determine whether a selective change in biliary cholesterol could be associated with regulation of GB SR-BI expression, a new set of experiments were carried out feeding mice with the same diets for a shorter time period. When C57BL/6J mice were fed a lithogenic diet for only 10 days, GB bile cholesterol concentration increased markedly, without significant changes in biliary bile salts or phospholipid content. ${ }^{30}$ Under this experimental condition, a clear downregulation of SR-BI was found (figs 4, 5). However, changes in bile salt composition are expected in lithogenic diet fed mice as dietary cholic acid is rapidly incorporated into the endogenous bile salt pool. Thus changes in bile acid composition apart from increased biliary cholesterol may also explain downregulation of GB SR-BI. To gain more insight into the specific bile component associated with changes in GB SR-BI expression, we performed a diosgenin feeding experiment in mice. Diosgenin is a plant steroid that selectively increases biliary cholesterol secretion but does not affect biliary secretion of bile salts and phospholipids or bile salt pool composition. ${ }^{36}$ Increased GB bile cholesterol content under a diosgenin supplemented diet also correlated with significant downregulation of SR-BI expression in GB epithelial cells. If bile is the source of cholesterol that regulates SR-BI expression in GBEC, our results may be explained by cholesterol dependent modulation of the transcriptional rate of the murine sr-bi gene. In fact, it has been shown that the promoter region of the SR-BI gene contains sterol response elements through which sterol regulatory element binding proteins can bind and thereby regulate transcription of this gene. ${ }^{41}$ Additional studies are required using polarised monolayer GBEC in cultured conditions to gain more insights into the mechanisms by which SR-BI expression might be regulated by cholesterol in GBECs.

This study was not aimed at directly evaluating the role of SR-BI in biliary lipid absorption in the GB. However, we performed additional experiments to test if GB SR-BI expression could be relevant in controlling GB wall cholesterol content and gall stone formation as an indirect measurement of a functional role of SR-BI expressed in the GB. Under lithogenic diet feeding, GB wall cholesterol content significantly decreased as early as three or six days and this change correlated with downregulation of SR-BI expression levels until day 12. However, after 30 days under a lithogenic diet, the cholesterol content of the GB wall intriguingly returned to basal levels while SR-BI expression levels remained low. These results suggest that SR-BI expression is not always functionally linked to control of GB wall cholesterol content. Alternatively, SR-BI downregulation could be involved in controlling GB cholesterol content but compensatory mechanism(s) may overcome this effect when a lithogenic diet is maintained for longer periods of time. However, the fact that SR-BI knockout mice showed similar GB wall cholesterol content under chow or lithogenic diets in comparison with wild-type mice strongly suggests that SR-BI expression in the GB epithelium does not play a major role in controlling GB wall cholesterol content. Moreover, this study clearly demonstrated that SR-BI expression is not essential for cholesterol gall stone formation under exogenous cholesterol supply with a standard lithogenic diet. Recent data published during the preparation of this manuscript also indicated that SR-BI is not critical in controlling either the formation of lithogenic bile or formation of gall stones. $^{42}$

To the best of our knowledge, no prior studies on the cholesterol content of the entire GB wall under lithogenic states have been reported. Studies performed in human and prairie dog GB have specifically shown increased cholesterol content in the plasma membrane of isolated GB muscle cells. ${ }^{14}{ }^{15}$ We were unable to detect an increment in total cholesterol content in the GB wall of mice, even 30 days after a lithogenic diet. However, changes in the cholesterol content of subcellular GB wall compartments or in net cholesterol flux through the GB wall cannot be excluded based on these studies.
In conclusion, we have demonstrated the presence of SR-BI expression in the apical membranes of human and mice GBECs. In the murine GBEC, SR-BI was downregulated in association with increased levels of GB biliary cholesterol. However, SR-BI expression in GB epithelial cells was not critical in controlling the cholesterol content of the GB wall or gall stone formation under a lithogenic diet. Further studies are required to elucidate the regulation of SR-BI in the GB and its function in GB physiology, particularly in transepithelial cholesterol transport as well as its potential role in prevalent GB disease conditions such as cholesterol gall stones and cholesterolosis.

\section{ACKNOWLEDGEMENTS}

This study was supported by grant FONDECYT (Fondo Nacional de Desarrollo Científico y Tecnológico) 8990006 to Attilio Rigotti and Juan F Miquel and grant 7980016 to Attilio Rigotti. We thank Dr Ann Hubbard and Susan Acton for providing anti- aminopeptidase-N and anti-SR-BI antibodies, respectively. We also thank Monty Krieger for sharing SR-BI knockout mice. We are indebted to Leonardo Andrade for excellent technical assistance in immunohistochemical assays, and Dr Marco Arrese for critical review of the manuscript.

\section{Authors' affiliations}

J F Miquel, M Moreno, L Amigo, H Molina, P Mardones, A Rigotti, Departamento de Gastroenterología, Facultad de Medicina, Pontificia Universidad Católica, Santiago, Chile

I I Wistuba, Departamento de Anatomía Patológica, Facultad de Medicina, Pontificia Universidad Católica, Santiago, Chile

\section{REFERENCES}

1 Apstein MD, Carey MC. Pathogenesis of cholesterol gallstones: a parsimonious hypothesis. Eur J Clin Invest 1996;26:343-52.

2 Shiffman MI, Sugerman HJ, Moore EW. Human gallbladder mucosal function. Gastroenterology 1990: 99:1452-9.

3 Giannini Corradini S, Yamashita G, Nuutinen H, et al. Human gallbladder mucosal function. Effects on intraluminal fluid and lipid composition in health and disease. Dig Dis Sci 1998; 43:335-43.

4 Das JB, Poulos ND, Ansari GG. Biliary lipid composition and bile acid profile during and after enteral fast of total parenteral nutrition in the rabbit. J Pediatric Gastroenterol Nutr 1996; 22:85-91.

5 Ostrow JD. Absorption by the gallbladder of bile salts, sulfobromophtalein, and iodipamide. J Lab Clin Med 1969;74:482-94. 6 Neiderhiser DH, Harmon CK, Roth HP. Absorption of cholesterol by the gallbladder. J Lipid Res 1976;17:1 17-24.

7 Neiderhiser DH, Morningstar WA, Roth HP. Absorption of lecithin and lysolecithin by the gallbladder. J Lab Clin Med 1973:82:891-7.

8 Jacyna MR, Ross PE, Bakar MA, et al. Characteristics of cholesterol absorption by human gallbladder: relevance to cholesterolosis. J Clin absorption by human gall
Pathol 1987:40:524-9.

9 Rathol 1987; 40:524-9. gallbladder. J Clin Pathol 1990;43:572-5.

10 Hayashi A, Lee S. Bidirectional transport of cholesterol between gallbladder epithelial cell and model bile. Am J Physiol 1996:271:G410-14.

11 Corradini GS, Ripani C, Della Guardia P, et al. The human gallbladder increases cholesterol solubility in bile by differential lipid absorption: A study using a new in vitro model of isolated intra-arterially perfused gallbladder. Hepatology 1998;28:314-22.

12 Einarsson C. Lipid absorption by the human gallbladder. Ital J Gastroenterol Hepatol 1999;31:571-3.

13 Corradini SG, Elisei W, Giovanelli L, et al. Impaired human gallbladder lipid absorption in cholesterol gallstone disease and its effect on cholesterol solubility in bile. Gastroenterology 2000;1 18:912-20.

14 Xiao ZL, Chen Q, Amaral J, et al. CCK receptor dysfunction in muscle membrane from human gallbladders with cholesterol stones. Am J Physio 1999;276:G1401-7.

15 Yu P, Chen P, Biancani $P$, et al. Membrane cholesterol alters gallbladder muscle contractility in prairie dogs. Am J Physiol 1996;271:G56-61.

16 Ostlund RE Jr, Bosner MS, Stenson WF. Cholesterol absorption efficiency declines at moderate dietary doses in normal human subjects. $J$ Lipid Res 1999;40:1453-8.

17 Jolley ChD, Dietschy JM, Turley SD. Genetic differences in cholesterol absorption in 129/Sv and C57BL/6 mice: effect on cholesterol responsiveness. Am J Physiol 1999;276:G1117-24.

18 Thurnhofer $\mathbf{H}$, Hauser $\mathrm{H}$. Uptake of cholesterol by small intestinal brush border membrane is protein-mediated. Biochemistry 1990;29:2142-8.

19 Berger KE, Tian H, Graf GA, et al. Accumulation of dietary cholesterol in sitisterolemia caused by mutations in adjacent $A B C$ transporters. Science 2000:290:1771-5.

20 Acton S, Rigotti A, Landschulz KT, et al. Identification of scavenger receptor SR-BI as a high density lipoprotein receptor. Science 1996;271:518-20. 
21 Rigotti A, Trigatti B, Babitt J, et al. Scavenger receptor BI-a cell surface receptor for high density lipoprotein. Curr Opin Lipidiol 1997:8:181-8.

22 Krieger M. Charting the fate of the "good cholesterol": identification and characterization of the HDL receptor SR-BI. Annu Rev Biochem 1999;68:523-58.

23 Krieger $\boldsymbol{M}$. Scavenger receptor class B type I is a multiligand HDL receptor that influences diverse physiologic systems. J Clin Invest 2001:108:793-7.

24 Hauser $\mathbf{H}$, Dyer JH, Nandy A, et al. Identification of a receptor mediating absorption of dietary cholesterol in the intestine. Biochemistry 1998; $37: 17843-50$

25 Voshol PJ, Schwarz M, Rigotti A, et al. Down-regulation of intestinal scavenger receptor class $B$, type I (SR-BI) expression in rodents under conditions of deficient bile delivery to the intestine. Biochem J 2001;356:317-25

26 Mardones P, Quinones V, Amigo L, et al. Hepatic cholesterol and bile acid metabolism and intestinal cholesterol absorption in scavenger receptor class B type I-deficient mice. J Lipid Res 2001;42:170-80.

27 Altmann SW, Davis HR, Yao X, et al. The identification of intestinal scavenger receptor class B, type I (SR-BI) by expression cloning and its
role in cholesterol absorption. Biochim Biophys Acta 2002;1580:77-93.

28 Johnson MSC, Svensson PA, Borén J, et al. Expression of scavenger receptor class B type I in gallbladder columnar epithelium. J Gastroenterol Hepatol 2002;17:713-20.

29 Auth MKH, Keitzer RA, Scholz M, et al. Establishment and immunological characterization of cultured human gallbladder epithelial cells. Hepatology 1993;18:546-55.

30 Amigo L, Quiñones V, Mardones P, et al. Impaired biliary cholesterol secretion and decreased gallstone formation in high cholesterol diet-fed apolipoprotein E-deficient mice. Gastroenterology 2000;1 18:1-9.

31 Hubbard AL. Targeting of membrane and secretory proteins to the apical domain in epithelial cells. Semin Cell Biol 1991;2:365-74.

32 Nuñez L, Amigo L, Mingrone G, et al. Biliary aminopeptidase-N and cholesterol crystallization defect in cholelithiasis. Gut 1995;37:422-6.
33 Rigotti A, Trigatti BL, Penman $M$, et al. A targeted mutation in the murine gene encoding the high density lipoprotein (HDL) receptor scavenger receptor class B type I reveals its key role in HDL metabolism. Proc Natl Acad Sci U S A 1997;94:12610-15.

34 Wilson MD, Rudel LL. Review of cholesterol absorption with emphasis on dietary and biliary cholesterol. J Lipid Res 1994;35:943-55.

35 Sehayek E, Ono JG, Shefer S, et al. Biliary cholesterol excretion: A novel mechanism that regulates dietary cholesterol absorption. Proc Natl Acad Sci U S A 1998;95:10194-9.

36 Nervi F, Marinovic I, Rigotti A, et al. Regulation of biliary cholesterol secretion. Functional relationship between the canalicular and sinusoidal cholesterol secretory pathways in the rat. J Clin Invest 1988;82:181825.

37 Kozarsky KF, Donahee MH, Rigotti A, et al. Overexpression of the HDL receptor SR-BI alters plasma HDL and bile cholesterol levels. Nature 1997;387:414-17

38 Dietschy JM, Turley SD, Spady DK. Role of the liver in the maintenance of cholesterol and low density lipoprotein homeostasis in different animal species, including humans. J Lipid Res 1993;34:1637-59.

39 Secknus R, Darby GH, Chernosky A, et al. Apolipoprotein A-I in bile inhibits cholesterol crystallization and modifies transcellular lipid transfer through cultured human gall-bladder epithelial cells. J Gastroent Hepatol 1999; 14:446-56.

40 Lafont $\mathbf{H}$, Domingo N, Groen A, et al. APF/CBP, the small, amphipathic, anionic protein(s) in bile and gallstones, consist of lipid-binding and calcium-binding forms. Hepatology 1997;25:1054-63.

41 Cao G, Garcia CK, Wyne KL, et al. Structure and localization of the human gene encoding SR-BI/CLA-1. Evidence for transcriptional control by steroidogenic factor 1. J Biol Chem 1997;272:33068-76.

42 Wang DQH, Carey MC. Susceptibility to murine cholesterol gallstone formation is not affected by partial disruption of the HDL receptor SR-BI. Biochem Biophy Acta 2002;1583:141-50.

For just US\$25 you can have instant access to the whole website for 30 days. During this time you will be able to access the full text for all issues (including supplements) available. You will also be able to download and print any relevant pdf files for personal use, and take advantage of all the special features Gut online has to offer.

\section{www.gutjnl.com}

